\title{
Processing of Temporal Unpredictability in Human and Animal Amygdala
}

\author{
Cyril Herry, ${ }^{1 *}$ Dominik R. Bach, ${ }^{2 *}$ Fabrizio Esposito, ${ }^{3}$ Francesco Di Salle, ${ }^{4,5}$ Walter J. Perrig, ${ }^{6}$ Klaus Scheffler, \\ Andreas Lüthi, ${ }^{1}$ and Erich Seifritz ${ }^{2,8}$ \\ ${ }^{1}$ Friedrich Miescher Institute for Biomedical Research, 4058 Basel, Switzerland, ${ }^{2}$ University Hospital of Psychiatry Bern, 3000 Bern, Switzerland, \\ ${ }^{3}$ Department of Neurological Sciences, University of Naples Federico II, 80127 Naples, Italy, ${ }^{4}$ Department of Neuroscience, University of Pisa, 56126 Pisa, \\ Italy, ${ }^{5}$ Department of Cognitive Neuroscience, University of Maastricht, 6200 Maastricht, The Netherlands, ${ }^{6}$ Institute of Psychology, University of Bern, \\ 3000 Bern, Switzerland, ${ }^{7}$ MR Physics, Department of Medical Radiology, University of Basel, 4031 Basel, Switzerland, and ${ }^{8}$ Department of Psychiatry, \\ University of Basel, 4025 Basel, Switzerland
}

The amygdala has been studied extensively for its critical role in associative fear conditioning in animals and humans. Noxious stimuli, such as those used for fear conditioning, are most effective in eliciting behavioral responses and amygdala activation when experienced in an unpredictable manner. Here, we show, using a translational approach in mice and humans, that unpredictability per se without interaction with motivational information is sufficient to induce sustained neural activity in the amygdala and to elicit anxiety-like behavior. Exposing mice to mere temporal unpredictability within a time series of neutral sound pulses in an otherwise neutral sensory environment increased expression of the immediate-early gene c-fos and prevented rapid habituation of single neuron activity in the basolateral amygdala. At the behavioral level, unpredictable, but not predictable, auditory stimulation induced avoidance and anxietylike behavior. In humans, functional magnetic resonance imaging revealed that temporal unpredictably causes sustained neural activity in amygdala and anxiety-like behavior as quantified by enhanced attention toward emotional faces. Our findings show that unpredictability per se is an important feature of the sensory environment influencing habituation of neuronal activity in amygdala and emotional behavior and indicate that regulation of amygdala habituation represents an evolutionary-conserved mechanism for adapting behavior in anticipation of temporally unpredictable events.

Key words: unpredictability; amygdala; anxiety; avoidance; habituation; fMRI

\section{Introduction}

The operation of predicting sensory information in time is fundamental for an organism to successfully interact with the environment and to maintain safety. This is, for example, substantiated by the finding that aversive or noxious stimuli, such as electric shocks or pain, produce more anxiety and fear if they are presented in a temporally unpredictable rather than a predictable way (Mineka and Kihlstrom, 1978; Grillon et al., 2004). This effect is accompanied by greater activation of the amygdala (Bornhovd et al., 2002), a core structure in the fear system of the mammalian brain (Weiskrantz, 1956; Büchel and Dolan, 2000; LeDoux, 2000; Davis and Whalen, 2001), and has been interpreted to reflect the processing of ambiguity (Whalen, 1998) or

Received Dec. 2, 2006; revised April 26, 2007; accepted April 26, 2007.

This work was supported by a grant from the Swiss National Science Foundation (63-58040.99/PP00B-103012, PP00B-68783) and by the Novartis Research Foundation. We thank Drs. P. Caroni, D. Paré, R. Paz, and D. De Quervain for comments on this manuscript and Dr. M. Schmidt-Daffy for providing the face stimuli.

${ }^{*}$ C.H. and D.R.B. contributed equally to this work.

Correspondence should be addressed to either of the following: Dr.Andreas Lüthi, Friedrich Miescher Institute for Biomedical Research, Maulbeerstrasse 66, CH-4058 Basel, Switzerland, E-mail: andreas.luthi@fmi.ch; or Dr. Erich Seifritz, University Hospital of Psychiatry Bern, Bolligenstrasse 111, CH-3000 Bern, Switzerland, E-mail: seifritz@puk.unibe.ch.

DOI:10.1523/JNEUROSCI.5218-06.2007

Copyright $\odot 2007$ Society for Neuroscience $\quad$ 0270-6474/07/275958-09\$15.00/0 uncertainty (Phelps and LeDoux, 2005; Rosen and Donley, 2006). However, the fundamental question whether unpredictability, as such, has anxiogenic properties and involves the amygdala has not been addressed so far, because unpredictability has not been studied per se but only in the context of unexpected or surprising emotionally salient stimulation including punishment (Grillon et al., 2006), reward or reward-predicting stimuli (Holland and Gallagher, 1993; Fiorillo et al., 2003; Paz et al., 2006), or facial expressions of fear (Adolphs et al., 1996; Whalen et al., 2001). Here, we directly tested this hypothesis and asked whether the amygdala is central in processing sensory information that does not allow an exact prediction in time. To test this hypothesis, we created a sensory environment that allowed us to study behavior and neural responses during temporal unpredictability or predictability within streams of neutral sensory events. Starting from predictable sound pulse sequences with regular pulse timing, we constructed temporally unpredictable sound pulse series by randomly varying the time interval between consecutive sound pulses (see Fig. 1). Using a translational approach in mice and humans, we found that predictable and unpredictable sound pulse series induce differential habituation of amygdala responses. Accordingly, temporal unpredictability promoted amygdala-dependent behaviors in mice and humans. 


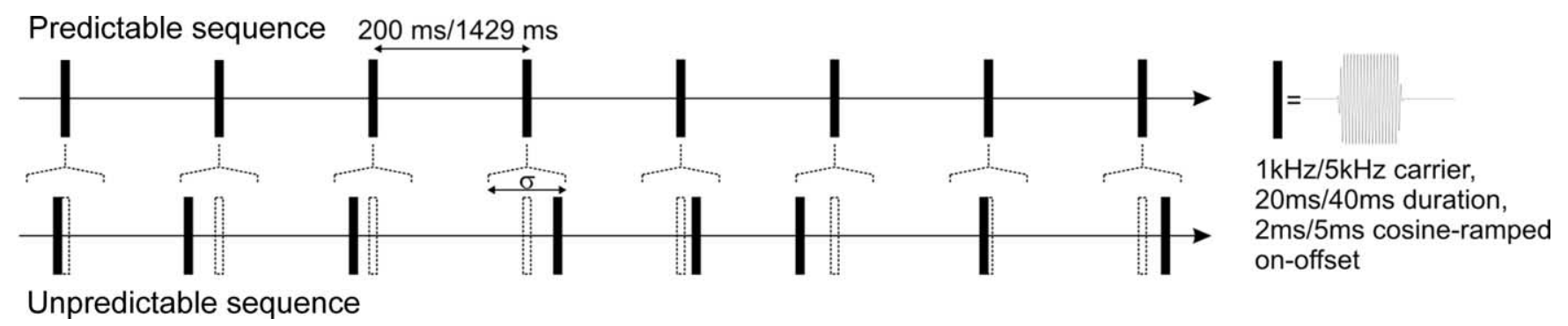

Figure 1. Schematic of auditory stimulation time series with predictable and unpredictable timing of the sound pulses. The randomized pulse sequence (bottom) was generated from the regular sequence (top) by a random temporal shift of each single pulse. The randomly selected temporal shift is confined to an interval $\sigma$ with uniform probability within $\sigma$ (see Materials and Methods for details).

\section{Materials and Methods \\ Sound pulse sequences}

Unpredictable sound pulse sequences were constructed by applying a random jitter of timing the sound pulses. In detail, the sound pulse time series were derived by randomly varying pulse timing within defined temporal boundaries of predictable sound pulse sequences with regular pulse timing as illustrated in Figure 1. The randomized pulse sequence (bottom) was generated from the predictable sequence (top) by a random temporal shift of each single pulse. The randomly selected temporal shift was confined to an interval $\sigma$ with uniform probability within $\sigma$. For experiments in humans, the carrier frequency was $1 \mathrm{kHz}$ with pulse duration of $40 \mathrm{~ms}$ and mean pulse spacing of $200 \mathrm{~ms}$ ( $5 \mathrm{~Hz}$ pulse repetition rate). In the unpredictable sequence, $\sigma$ was set to $120 \mathrm{~ms}$; in the predictable sequence, $\sigma$ was set to $0 \mathrm{~ms}$. For animal experiments, the carrier frequency was increased to $5 \mathrm{kHz}$ with a pulse duration of $20 \mathrm{~ms}$ and mean pulse spacing of $200 \mathrm{~ms}(5 \mathrm{~Hz})$ or $1429 \mathrm{~ms}(0.7 \mathrm{~Hz})$. $\sigma$ was set to 140 or $1368 \mathrm{~ms}$ for the unpredictable sequence, and $\sigma$ was $0 \mathrm{~ms}$ for the predictable sequence. For all pulse sequence variants, the sound pulse was switched on and off smoothly using cosine-shaped increasing and decreasing ramps with a duration of $2 \mathrm{~ms}$ (for $20 \mathrm{~ms}$ pulse duration) or 5 $\mathrm{ms}$ (for $40 \mathrm{~ms}$ pulse duration).

\section{Animals}

Male C57BL6/J mice (3 months old; RCC, Füllinsdorf, Switzerland) were housed individually for $7 \mathrm{~d}$ before all experiments, under a $12 \mathrm{~h} \mathrm{light/}$ dark cycle, and provided with food and water ad libitum. All animal procedures were executed in accordance with institutional guidelines and were approved by the Veterinary Department of the Canton of Basel-Stadt.

\section{Immunocytochemistry}

Naive mice were placed in an open field consisting of a circular transparent Plexiglas box ( $27 \mathrm{~cm}$ diameter, $80 \mathrm{~cm}$ high) placed inside a soundattenuating and temperature-regulated cubicle and exposed to $2 \mathrm{~min}$ predictable (carrier frequency, $5 \mathrm{kHz}$; pulse duration, $20 \mathrm{~ms}$; repetition rate, $5 \mathrm{~Hz} ; \sigma, 0 \mathrm{~ms}$; sound level, $85 \mathrm{~dB}$ ) or unpredictable (same as predictable sound, except for random pulse timing within $\sigma, 140 \mathrm{~ms}$ ) sound pulse sequence stimulation. Mice were perfused transcardially with icecold $4 \%$ paraformaldehyde in $0.1 \mathrm{M}$ PBS 30 or $120 \mathrm{~min}$ after the beginning of sound pulse sequence stimulation. Brains were prepared for immunocytochemistry using a primary polyclonal rabbit anti-c-Fos antibody [anti-c-Fos (Ab-5) (4-17) rabbit polyclonal antibody, PC38; 1:20,000 dilution; Oncogene Research Products, San Diego, CA] as described previously (Herry and Mons, 2004). A biotinylated goat antirabbit antibody (1:2000 in PBS; Jackson ImmunoResearch, West Grove, PA) was used as a secondary antibody. Staining was revealed using the avidin-biotin peroxidase method (Vectastain Elite kit; Vector Laboratories, Burlingame, CA) coupled to diaminobenzidine as the chromogen. Stained slices were imaged at $20 \times$ using a Nikon (Tokyo, Japan) DX1200 camera mounted on a Nikon Eclipse E600 microscope. Quantitative analysis of c-Fos-positive nuclei was performed using a computerized image analysis system (Visiolab 2000 version 4.50; Biocom, LesUlis, France). Structures were defined according to Franklin and Paxinos (1997). Immunoreactive neurons were counted bilaterally using a mini- mum of three sections. To account for differences in background staining and detection thresholds between experimental series, results were averaged across animals and expressed as a percentage of counts in animals exposed to predictable sound pulse sequence stimulations in the same experimental series. Statistical analyses were performed using Scheffe's $F$ test post hoc comparisons at the $p<0.05$ level of significance. Results are presented as mean \pm SEM.

\section{Surgery and electrophysiology}

Mice were anesthetized with isoflurane (induction, 5\%; maintenance, $2.5 \%$ ) in $\mathrm{O}_{2}$. Body temperature was maintained with a heating pad. Mice were secured in a stereotaxic frame and unilaterally implanted with a multiwire electrode aimed at the following coordinates according to Franklin and Paxinos (1997): $1.7 \mathrm{~mm}$ posterior to bregma, $\pm 3.1 \mathrm{~mm}$ lateral to midline, and $4-4.3 \mathrm{~mm}$ deep from the cortical surface. The electrodes consisted of eight individually insulated nichrome wires (13 $\mu \mathrm{m}$ diameter; impedance, 1-3 M $\Omega$; California Fine Wire, Grover Beach, $\mathrm{CA})$ contained in a 26 gauge stainless steel guide cannula. The wires were attached to a 10-pin connector (Omnetics, Minneapolis, MN). The implant was secured using dental cement (Heraeus Kulzer, Hanau, Germany). After surgery, mice were allowed to recover for $7 \mathrm{~d}$. Topical analgesia was applied for $3 \mathrm{~d}$. Electrodes were connected to a headstage (Plexon, Dallas, TX) containing eight unity-gain operational amplifiers. The headstage was connected to an eight-channel, computer-controlled preamplifier via a rotating commutator (Plexon). Neuronal activity was digitized at $40 \mathrm{kHz}$ and isolated by time-amplitude window discrimination and template matching using a Multichannel Acquisition Processor system (Plexon). Naive mice ( $n=8)$ were placed in an open field consisting of a circular transparent Plexiglas box $(27 \mathrm{~cm}$ diameter, $80 \mathrm{~cm}$ high) placed inside a sound-attenuating and temperature-regulated cubicle. After a $2 \mathrm{~min}$ habituation period, mice were submitted to predictable (carrier frequency, $5 \mathrm{kHz}$; pulse duration, $20 \mathrm{~ms}$; repetition rate, 0.7 $\mathrm{Hz} ; \sigma, 0 \mathrm{~ms}$; sound level, $85 \mathrm{~dB}$ ) and unpredictable (same as predictable sound, except for random pulse timing within $\sigma, 1368 \mathrm{~ms}$ ) sound pulse sequence stimulation. To avoid rapid habituation of sound pulse-evoked neural activity, sound pulses were delivered at an average repetition rate of $0.7 \mathrm{~Hz}$. Stimulation patterns were delivered according to a crossover protocol balanced across subjects. Tone-induced neural activity was calculated by comparing the firing rate during the first $500 \mathrm{~ms}$ after sound pulse onset with the firing rate recorded during the $500 \mathrm{~ms}$ before sound pulse onset using a $Z$-score transformation. Of 38 cells recorded, only those displaying significant $Z$-score changes of at least \pm 1 during the first $100 \mathrm{~ms}$ after sound pulse onset (predictable or unpredictable sound pulse sequence) were included in the analysis (28 tone-responsive cells). At the conclusion of the experiment, recording sites were marked with electrolytic lesions before perfusion, and electrode locations were reconstructed with standard histological techniques. Statistical analyses were performed using paired $t$ test or repeated-measures ANOVA, followed by Scheffe's $F$ test post hoc comparisons at the $p<0.05$ level of significance. Results are presented as mean \pm SEM.

\section{Animal behavior}

Elevated plus-maze. The elevated plus-maze (EPM) consisted of a center platform and four arms placed $50 \mathrm{~cm}$ above the floor. Two arms were 
enclosed within walls (closed arms), and the two others had low rims (open arms). Naive mice were placed in the center of the maze for a $5 \mathrm{~min}$ silent period, after which they were exposed to $5 \mathrm{~min}$ of predictable sound pulse sequence stimulation (carrier frequency, $5 \mathrm{kHz}$; pulse duration, $20 \mathrm{~ms}$; repetition rate, $5 \mathrm{~Hz} ; \sigma, 0 \mathrm{~ms}$; sound level, $85 \mathrm{~dB}$ ) or to $5 \mathrm{~min}$ of unpredictable (same as predictable sound, except for random pulse timing $\sigma, 140 \mathrm{~ms}$ ) sound pulse sequence stimulation (speaker located above the maze). The time spent in the different compartments (closed vs open arms), and the number of arm entries was scored using a videobased tracking system (EthoVision System; Noldus, Wageningen, The Netherlands). Statistical analyses were performed using paired or unpaired $t$ test comparisons at the $p<0.05$ level of significance. Results are presented as mean \pm SEM.

Locomotor activity. Naive mice were placed in an open field consisting of a circular transparent Plexiglas box $(27 \mathrm{~cm}$ diameter, $80 \mathrm{~cm}$ high) placed inside a sound-attenuating and temperature-regulated cubicle. A speaker was positioned on the top of the cubicle to deliver predictable (carrier frequency, $5 \mathrm{kHz}$; pulse duration, $20 \mathrm{~ms}$; repetition rate, $5 \mathrm{~Hz} ; \sigma$, $0 \mathrm{~ms}$; sound level, $85 \mathrm{~dB}$ ) or unpredictable (same as predictable sound, except for random pulse timing within $\sigma, 140 \mathrm{~ms}$ ) sound pulse sequences. After a $2 \mathrm{~min}$ habituation period, mice were exposed for $2 \mathrm{~min}$ to predictable sound pulse sequence stimulation and, 5 min later, for 2 min to unpredictable sound pulse sequence stimulation. General locomotor activity was assessed by measuring the mean speed (centimeters per second) during sound pulse sequence stimulation using an automatic infrared beam detection system placed on the bottom of the experimental chamber (Coulbourn Instruments, Allentown, PA). Statistical analyses were performed using paired $t$ test comparisons at the $p<0.05$ level of significance. Results are presented as mean \pm SEM.

Place avoidance. The place avoidance apparatus consisted of a gray Plexiglas square chamber $(38.5 \times 25 \times 25 \mathrm{~cm})$ and a gray Plexiglas circular chamber ( $35 \mathrm{~cm}$ diameter), both of which were connected to one another by a small gray square-shaped Plexigas alleyway $(10 \times 10 \times 25$ $\mathrm{cm})$. Geometric cues were placed on the wall of the circular chamber only. After each trial, the square and circular chambers were cleaned with $1 \%$ acetic acid or $70 \%$ ethanol, respectively. The entire experiment was continuously monitored using a video camera-based system (EthoVision System; Noldus). On the first day, mice were placed in front of a wall in the central alleyway for two sessions of 15 min during which they could freely explore the apparatus. At the end of this phase, the compartment in which mice spent most of their time was designated as the preferred compartment. Mice were then divided into two groups and placed in the apparatus for a $15 \mathrm{~min}$ session on the next day. The first group was exposed to unpredictable sound pulse sequence stimulation (carrier frequency, $5 \mathrm{kHz}$; pulse duration, $20 \mathrm{~ms}$; repetition rate, $5 \mathrm{~Hz}$; random pulse timing within $\sigma, 140 \mathrm{~ms}$; sound level, $85 \mathrm{~dB}$ ) when the animals were in their preferred compartment and to predictable sound pulse sequence stimulation (same as unpredictable sound, except for regular pulse timing within $\sigma, 0 \mathrm{~ms}$ ) when the animals were in their nonpreferred compartment. The opposite protocol was done for the second group. Statistical analyses were performed using repeated-measures ANOVA, followed by Scheffe's $F$ test post hoc comparisons at the $p<0.05$ level of significance. Results are presented as mean \pm SEM.

\section{Functional imaging in humans}

We used functional magnetic resonance imaging (fMRI) to examine neural activity in healthy subjects ( 5 females and 15 males; age, $29.5 \pm 8.1$ years). Predictable (carrier frequency, $1 \mathrm{kHz}$; pulse duration, $40 \mathrm{~ms}$; repetition rate, $5 \mathrm{~Hz} ; \sigma, 0 \mathrm{~ms}$; sound level, $95 \mathrm{~dB}$ ) and unpredictable (same as predictable sound, except for random pulse timing within $\sigma, 120 \mathrm{~ms}$ ) sound pulse sequences were presented in $60 \mathrm{~s}$ stimulation blocks interrupted by $30 \mathrm{~s}$ silent blocks. The sequence was S-P-S-U-S-P-S-U-S (S denoting the periods of silence, $\mathrm{P}$ denoting the periods with predictably timed sound pulse stimulation, and $\mathrm{U}$ denoting the periods with unpredictably timed sound pulse stimulation) and was constant across subjects. Subjects were instructed to passively listen to the sounds but not to perform active tasks. Anatomical (three-dimensional magnetizationprepared rapid acquisition gradient-echo T1 sequence; resolution, 1 $\mathrm{mm}^{3}$ ) and functional images ( $865 \mathrm{~T} 2{ }^{*}$-weighted gradient-recalled echo- planar sequence; repetition time, $2140 \mathrm{~ms} ; 20$ contiguous oblique slices; thickness, $4 \mathrm{~mm}$; in-plane resolution, $2.8 \times 2.8 \mathrm{~mm}^{2}$; parallel and centered to lateral sulcus, covering the entire cerebrum except for the most apical regions extending $\sim 30 \mathrm{~mm}$ in $z$-direction) were acquired on a 1.5T Symphony scanner equipped with a Quantum (Durham, NC) gradient system and a circularly polarized head coil (Siemens, Erlangen, Germany). Preprocessing of the Talairach-transformed functional image time series included discharge of the first eight frames, resampling into 3 $\mathrm{mm}$ isotropic voxels, slice acquisition time correction by sinc interpolation, three-dimensional motion correction, and temporal and spatial filtering (high-pass, five cycles; low-pass, $2.8 \mathrm{~s}$; and $6 \mathrm{~mm}$ full-width half-maximum Gaussian kernel) using BrainVoyager QX 1.5 (Brain Innovation, Maastricht, The Netherlands). The variance of all image time series was estimated voxelwise according to a random-effects convolution-based general linear model (GLM) analysis (Friston et al., 1995). Predictors encoding the response to predictable and unpredictable sound pulse sequences against a silent baseline level were defined as box-car functions and convolved using a single-gamma function (Boynton et al., 1996) to account for hemodynamic response. For each subject and each voxel of the imaging slab, the four " $\beta$ " weights of the four predictors were estimated using a GLM fit-refit procedure to correct for residual serial correlation in the error terms according to a first-order autoregressive model (Bullmore et al., 1996). To draw population-level inferences from statistical maps, the $\beta$ estimates at each voxel together with their linear combinations (contrasts) entered appropriate $t$ tests with subjects as random observations (random-effects analysis). The resulting $t$ maps for the main effects of all stimulus responses as well as for the linear differential contrast balancing the unpredictable sound stimuli to the predictable sound stimulus for the "null hypothesis" were overlaid on the Talairach-transformed anatomical scan (averaged across study subjects). To localize the significant effects on the average anatomy, a threshold was applied to the $t$ maps, which protected against falsepositive effects at $5 \%$ (corrected for multiple comparisons). For the main effects of all responses, a whole-slab Bonferroni-corrected threshold of $p=0.05$ was accepted. For differential effects (unpredictable vs predictable), we used a combined voxel- and cluster-level correction approach based on the three-dimensional extension of the randomization procedure described by Forman et al. (1995) (see also Etkin et al., 2004). Accordingly, a voxel-level threshold was set at $t=3.85(p=0.001$, uncorrected); then, the thresholded maps were submitted to a whole-slab correction criterion based on the estimate of the spatial smoothness of the maps and on an iterative procedure (Monte Carlo simulation) for estimating cluster-level false-positive rates. After 1000 iterations, maps were applied the minimum cluster size threshold, which yielded a cluster-level false-positive rate $(\alpha)$ of $5 \%$ (note that this statistical approach does not use anatomically predefined areas for statistical inferences and is different from what is called "small volume correction"). To rule out that the studied effects were driven by habituating responses to prolonged passive stimulation (Breiter et al., 1996; Büchel et al., 1998; LaBar et al., 1998), we performed a second random-effects GLM analysis with a different set of predictors, modeling the hemodynamic response function separately for the first half (early-phase response) and the second half (late-phase response) of the stimulation interval, similar to previous work (Becerra et al., 2001; Schmahl et al., 2006). We considered the linear contrast of the early phase of the response to each unpredictable sound stimulus against the corresponding late phase, assuming that possible habituation effects would result in a relative suppression of the late-phase compared with the early-phase responses.

\section{Human behavior}

Affective preference of the unpredictable and predictable sound pulse sequence was examined in healthy subjects ( 20 females and 20 males; age, $23.6 \pm 4.8$ years), who were presented predictable (carrier frequency, 1 $\mathrm{kHz}$; pulse duration, $40 \mathrm{~ms}$; repetition rate, $5 \mathrm{~Hz} ; \sigma, 0 \mathrm{~ms}$; sound level, 85 $\mathrm{dB}$ ) and unpredictable (same as predictable sound, except for random pulse timing within $\sigma, 80 \mathrm{~ms}$ ) sound pulse sequences through headphones. They were asked which sound they found more unpleasant (nominal data were compared using the $\chi^{2}$ test). To examine the effects of unpredictably timed sound pulses on selective attention to facial 
threat, a visual dot probe task (Mogg et al., 1997, 2000; Bradley et al., 1999; Koster et al., 2004) was used (see Fig. 5c). Healthy subjects (18 females and 18 males; age, $22.6 \pm 3.7$ years) were presented on a liquid crystal display screen, consecutively a fixation cross $(950 \mathrm{~ms})$, two faces (50 ms; each face, $8^{\circ}$ visual angle; distance between the faces, $6^{\circ}$ ), and a dot $\left(0.25^{\circ}\right.$ visual angle $)$ appearing in place of one of the two faces $(1000$ $\mathrm{ms})$. The intertrial intervals varied randomly between 500 and $1500 \mathrm{~ms}$. The dot served as target of the space position of which subjects had to press a button (right or left), and reaction times (RTs) were measured. We used faces from the Ekman set (Ekman and Friesen, 1975; Mogg et al., 2000) selected and morphed to correspond for affect recognition performance and affect intensity as rated previously by a normative sample of healthy human subjects (Schmidt and Erdmann, 2003). Picture presentation consisted of pairs of a neutral and an angry face, or of pairs with two neutral faces. In neutral-angry face pairings, a trial was considered "congruent" (dot at angry face position) or "incongruent" (dot at neutral face position), and in neutral-neutral face pairings, all trials were considered "neutral." The task was divided into blocks of 48 trials ( 24 neutral, 12 congruent, 12 incongruent; length of each block, 219 s). They were repeated during headphone presentation of predictable (carrier frequency, $1 \mathrm{kHz}$; pulse duration, $40 \mathrm{~ms}$; repetition rate, $5 \mathrm{~Hz} ; \sigma, 0 \mathrm{~ms}$; sound level, $85 \mathrm{~dB}$ ) and unpredictable (same as predictable sound, except for random pulse timing within $\sigma, 80 \mathrm{~ms}$ ) sound sequence. Each condition was repeated twice according to a crossover protocol balanced across subjects. Analysis of covariance was used considering congruent and incongruent trials during predictable and unpredictable sounds as repeated measures and mean RTs from neutral trials as covariate. The bias index was calculated by subtracting mean congruent RTs from mean incongruent RTs.

\section{Results}

\section{Unpredictability induces sustained amygdala activation in mice}

We first addressed whether a stimulation sequence of unpredictably timed sound pulses activates amygdala in rodents as measured by the expression of the immediate-early gene c-fos. Although strong c-Fos induction has been described $1-2 \mathrm{~h}$ after sensory stimulation (Filipkowski et al., 2000; Bisler et al., 2002) or fear conditioning (Herry and Mons, 2004), cortical c-Fos levels can rise as early as $30 \mathrm{~min}$ after sensory stimulation (Chaudhuri et al., 2000). We thus analyzed c-Fos induction 30 and $120 \mathrm{~min}$ after the beginning of sound pulse stimulation. Whereas both unpredictably and predictably timed sound pulses induced a significant increase in c-Fos immunoreactivity compared with naive mice in the lateral (LA) and basal (BA) nuclei of the amygdala $(n=6$; $p<0.05$; data not shown), unpredictably compared with predictably timed sound pulses induced a significantly greater increase in c-Fos immunoreactivity in LA (at both time points) and BA (only at the 30 min time point) (Fig. 2). In contrast, c-Fos labeling was not significantly different between unpredictably and predictably stimulated animals in central amygdala, primary motor cortex, ventral hippocampus, ventral auditory cortex, and the medial division of the medial geniculate nucleus of the thalamus where auditory afferents to the amygdala originate (Fig. $2 a$ ).

Next, we directly examined the effect of predictably and unpredictably timed sound pulses on single-cell activity in LA and BA using in vivo single-unit recordings (Fig. $3 a$ ). A total of 38 cells were recorded, and only tone-responsive cells $(n=28)$ were included in the analysis. Because results obtained in LA and BA [together referred to as basolateral amygdala (BLA)] did not significantly differ from each other, the values were pooled. For each cell, the auditory pulse-evoked activity from 0 to $500 \mathrm{~ms}(20 \mathrm{~ms}$ bins) after pulse onset was quantified as an average $Z$ score (i.e., each bin expressed as the number of SDs above or below the mean baseline firing rate) during predictably and unpredictably timed sound pulse stimulation. The average $Z$ score of auditory pulseevoked activity was significantly greater during unpredictably timed stimulation (predictable, $0.49 \pm 0.29$; unpredictable, $1.57 \pm 0.41 ; n=28 ; p=0.007$, two-tailed paired $t$ test on the average $Z$ score of $0-80 \mathrm{~ms}$ after sound pulse onset) (Fig. $3 b-d)$, with no difference in the average spontaneous activity (measured during the $500 \mathrm{~ms}$ preceding each sound pulse; predictable, $1.34 \pm 0.27 \mathrm{~Hz}$; unpredictable, $1.22 \pm 0.27 \mathrm{~Hz}$; $\left.n=28 ; F_{(1,27)}=0.260 ; p=0.61\right)$. Thus, consistent with the c-Fos results, individual BLA neurons fire significantly more in response to unpredictably timed compared with predictably timed sound pulses.

Previous single-unit studies have shown that a substantial fraction of neurons in the BLA rapidly habituate in response to repetitive stimulation (Ben-Ari and Le Gal La Salle, 1974; Bordi and LeDoux, 1992; Quirk et al., 1997). To address whether differential habituation could account for the increased overall activity during unpredictably timed sound pulse stimulation, we analyzed the time course of sound pulse-evoked neural activity during stimulation sequences. Theoretically, at least three sound pulses are necessary to assess temporal predictability of a sequence. In keeping with this, neural activity evoked by the first two pulses did not differ between predictable and unpredictable stimulation sequences $\left(n=28 ; F_{(1,54)}=0.74 ; p=0.39\right.$; Scheffé's $F$ test). However, over time, BLA neurons habituated significantly more to predictably than to unpredictably timed sound pulses (Fig. 3e). Next, we examined whether the differential habituation of sound pulse-evoked activity as measured by the $Z$ score could be explained by changes in spontaneous activity over time. Whereas spontaneous activity remained constant during predictable sound pulse stimulation (ratio of spontaneous activity during the $500 \mathrm{~ms}$ before pulses $16-20$ /pulses $1-2,99 \pm 17 \%$; $n=28 ; F_{(4,108)}=1.015 ; p=0.40$, one way ANOVA with repeated measures), we found a significant reduction in spontaneous activity during unpredictably timed auditory stimulation (ratio of spontaneous activity during the $500 \mathrm{~ms}$ before pulses 16-20/ pulses $1-2,68 \pm 11 \% ; n=28 ; F_{(4,108)}=5.380 ; p<0.001$, oneway ANOVA with repeated measures). However, this reduction in spontaneous activity during unpredictably timed auditory stimulation had only a minor effect on $Z$-score habituation. Recalculating $Z$ scores for both predictable and unpredictable stimulation sequences using identical values for spontaneous activity confirmed that unpredictable auditory stimulation does not induce any significant habituation of pulse-evoked responses $(n=$ $28 ; F_{(4,108)}=0.321 ; p=0.86$; one-way ANOVA with repeated measures). Moreover, we did not find any evidence for anticipatory activity over the time course of predictable or unpredictable stimulation sequences (data not shown).

Finally, differential habituation of sound pulse-evoked responses could be explained by a disproportionate recovery from habituation during the longer than average interstimulus intervals occurring during unpredictable stimulation. This is unlikely to be the case because the comparison of subsets of stimuli with equal interstimulus intervals $(0.7 \mathrm{~Hz}$ vs $0.75 \pm 0.05 \mathrm{~Hz} ; n=11)$ revealed significantly stronger neural responses during unpredictably compared with predictably timed stimulation $(Z$ score $_{\text {unpredictable }} / Z$-score predictable, $2.8 \pm 0.6 ; n=11 ; p=0.01$ ). Thus, whereas predictable stimulation induces rapid habituation of auditory-evoked neuronal responses of BLA neurons, the same neurons switch to a nonhabituating mode when auditory stimulation is presented in an unpredictable manner. 


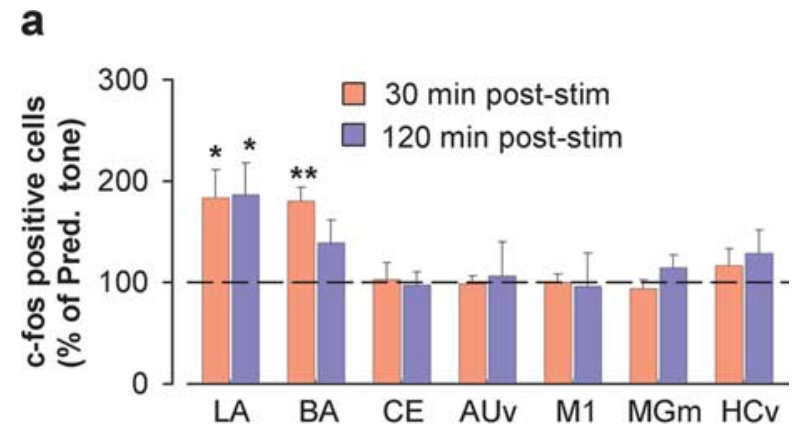

b
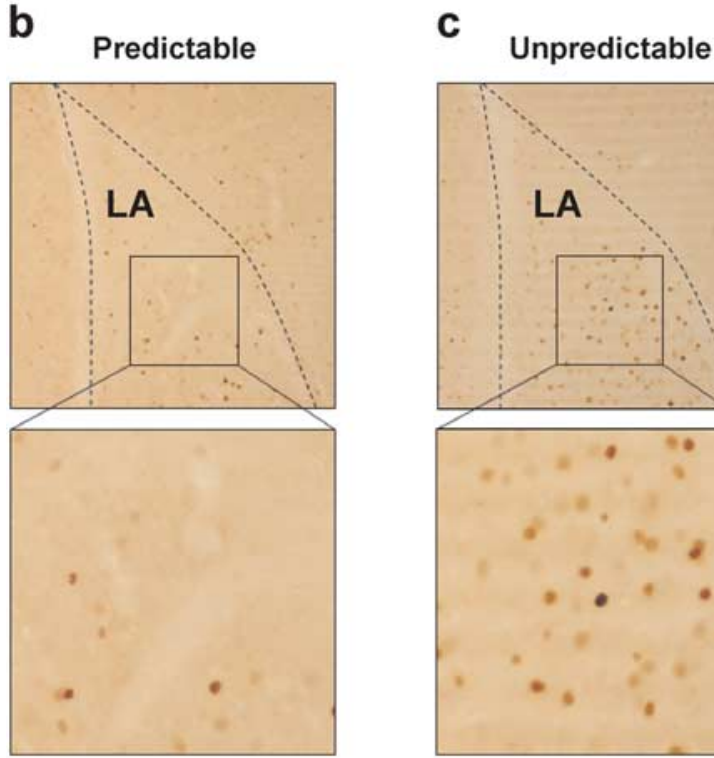

d

\section{Predictable}

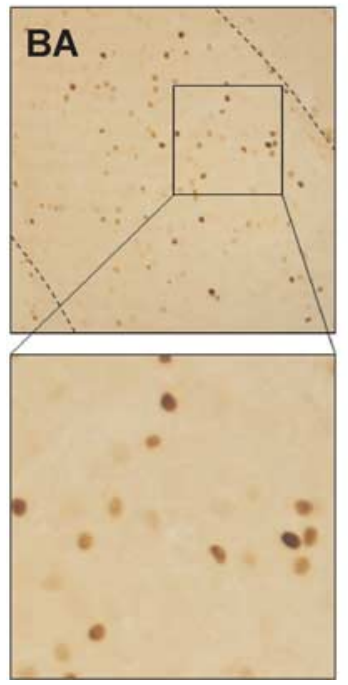

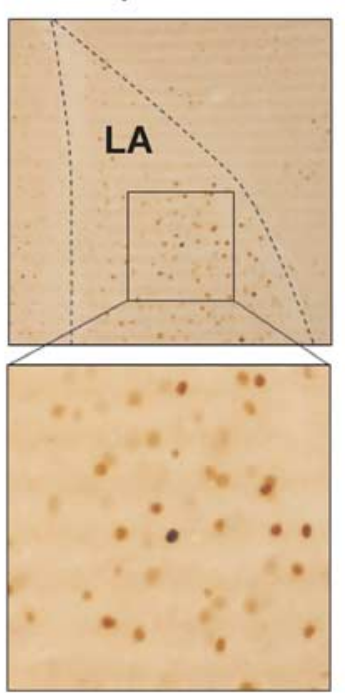

e
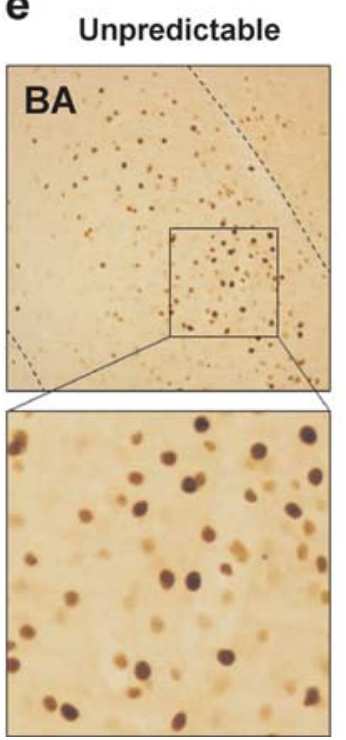

Figure 2. Increased c-Fos induction by unpredictably timed sound pulse stimulation in the lateral and basal amygdala. $\boldsymbol{a}, \mathrm{c}-\mathrm{F}$ os induction in the LA, BA, and central (CE) nuclei of the amygdala, ventral auditory cortex (AUv), medial division of the medial geniculate nucleus of the thalamus (MGm), ventral hippocampus (HCv), and primary motor cortex (M1) quantified 30 $\min$ ( $n=4-5$ per group) and $120 \min$ ( $n=4-6$ per group) after the start of unpredictably timed sound pulse stimulation (stim). Results are expressed as a percentage of the number of c-Fos-positive cells induced by predictably timed sound pulse stimulation (Pred. tone). All $p<$ 0.05 (Scheffé's F test). ${ }^{*} p<0.05 ;{ }^{* *} p<0.01$. b, c, Photomicrographs illustrating increased c-Fos levels in the LA 120 min after the start of unpredictably versus predictably timed sound pulse stimulation. $\boldsymbol{d}, \boldsymbol{e}$, Photomicrographs illustrating increased c-Fos levels in the BA $30 \mathrm{~min}$ after the start of unpredictably versus predictably timed sound pulse stimulation.

Unpredictability induces anxiety-like behavior and avoidance in mice

Because the BLA has been widely implicated in controlling emotional behavior (Weiskrantz, 1956; Büchel and Dolan, 2000; LeDoux, 2000; Davis and Whalen, 2001), we sought to investigate whether unpredictably timed sound pulse stimulation induces behavioral changes related to anxiety or aversion. BLA is thought to influence anxiety-like behavior on the EPM (Vyas and Chattarii, 2004), a standard paradigm for assessing anxiety in rodents (Lister, 1987). We thus placed mice on an EPM while being exposed to predictably or unpredictably timed sound pulse sequences for $5 \mathrm{~min}$. Notably, stimulation with predictably timed sound pulses did not induce any changes in anxiety-like behavior on the EPM relative to a silent control period (Fig. 4a). In contrast, mice spent less time in the open arms of the EPM when exposed to unpredictably timed sound pulses (Fig. 4a). This effect was not attributable to changes in general activity levels because we did not find any differential effect of predictably and unpredictably timed sound pulses on the number of total arm entries ( $n=5$ per group; $p=0.918$, unpaired $t$ test; data not shown) and on locomotor activity (Fig. $4 b$ ). This shows that whereas exposure to predictably timed auditory stimulation is not anxiogenic on the EPM, unpredictably timed auditory stimulation induces anxiety-like behavior in rodents.

Unpredictability might induce anxiety-like behavior because it is intrinsically aversive and thus anxiogenic. Alternatively, it might nonspecifically affect arousal, thereby causing heightened sensitivity to potential threats. If unpredictability is intrinsically anxiogenic and aversive, it should induce avoidance behavior. To address this, mice were allowed to freely explore a maze composed of two different compartments. After this habituation period, a preferred and nonpreferred compartment were identified based on the time spent in each compartment: mice spent significantly more time in their preferred compartment $(n=5 ; p<$ 0.05 , paired $t$ test; data not shown). Subsequently, sequences of unpredictably timed sound pulses were presented each time the mouse entered its preferred compartment, and predictably timed sounds were presented when it entered the nonpreferred compartment. As a consequence, mice started to progressively avoid their initially preferred compartment (Fig. $4 c$ ). When tested the next day in the absence of any auditory stimulation, mice still avoided the compartment in which they had been exposed to sequences with unpredictably timed sound pulses (no significant difference in time spent in the two compartments; $n=5 ; p=$ 0.50 , paired $t$ test; data not shown), confirming that behavioral consequences were not attributable to an unspecific effect on arousal. In contrast, the reverse experiment failed to reveal any significant shift in behavior (i.e., mice continued to spend more time in the preferred compartment if it was associated with predictably timed sound pulses) (Fig. 4c). Thus, in rodents, temporally unpredictable auditory stimulation is intrinsically aversive, produces sustained amygdala activation, and leads to increased anxiety-like behavior.

\section{Unpredictability induces sustained amygdala activation in humans}

To test whether amygdala activation induced by unpredictably timed sound pulses as observed in mice is also present in humans, we used fMRI with $1.5 \mathrm{~T}$ in 20 healthy subjects. Standard gradient echo-planar imaging on $1.5 \mathrm{~T}$ covering the entire brain except of the most cranial $30 \mathrm{~mm}$ was performed in 20 healthy subjects. A block-design ( $60 \mathrm{~s}$ on, $30 \mathrm{~s}$ off) experimental stimulation paradigm consisting of sequences with predictably versus unpredict- 
a
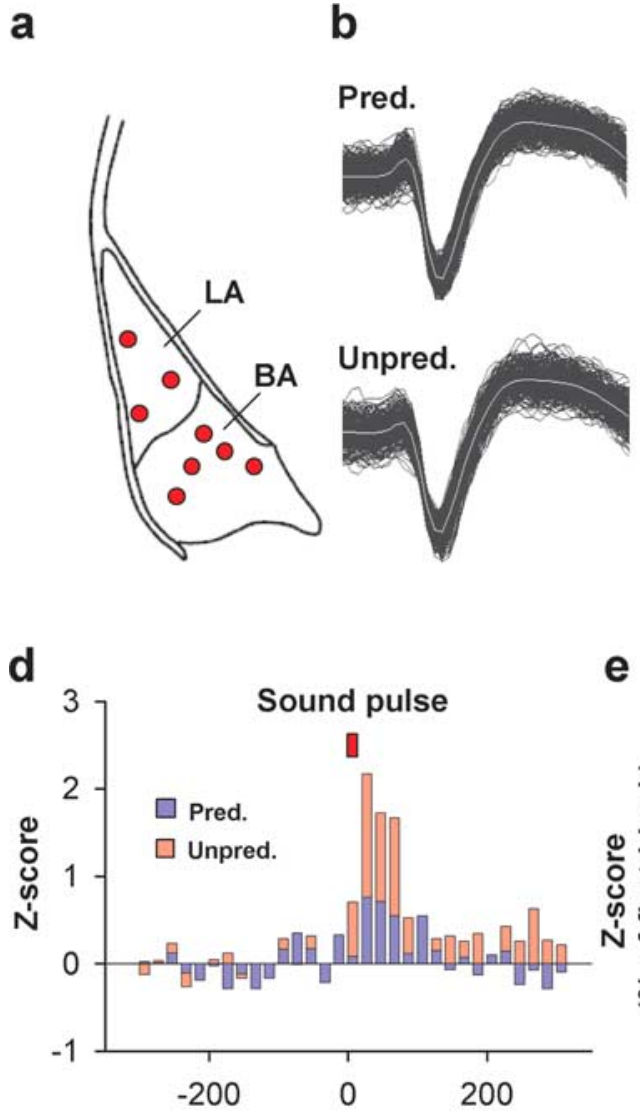

Time (ms)
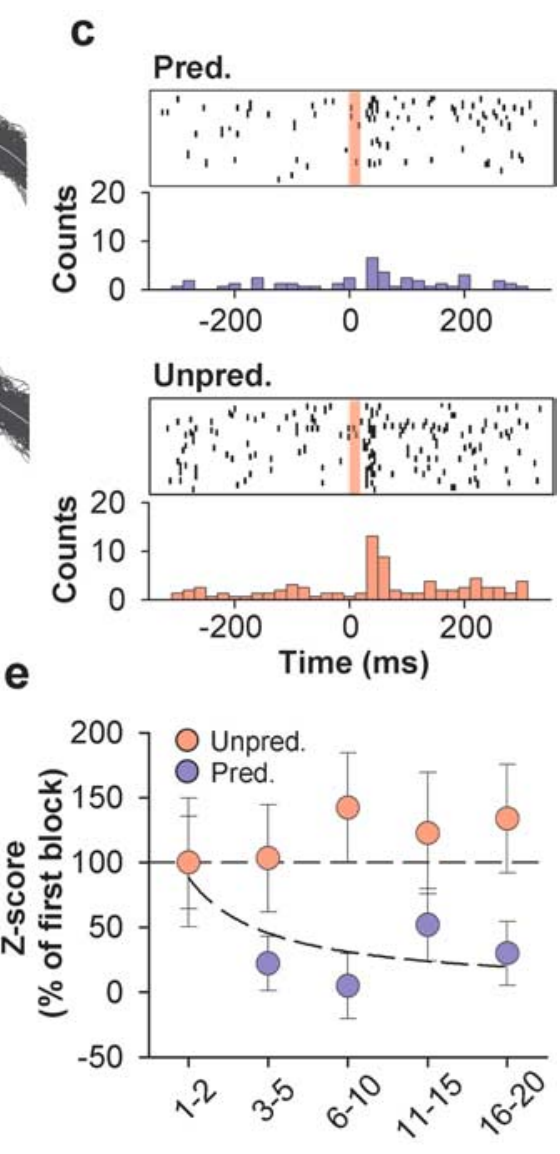

Sound pulse \#

Figure 3. Differential neural activity during unpredictably and predictably timed sound pulse stimulation in BLA neurons. $\boldsymbol{a}$, Recording sites in the BLA. $\boldsymbol{b}$, Waveforms of a representative neuron recorded during predictably and unpredictably timed sound pulse stimulation. c, Raster plot and peristimulus time histogram (PSTH) illustrating sound pulse-evoked activity of a representative neuron recorded in the BLA during predictably (top) and unpredictably (bottom) timed sound pulse stimulation (bins of 20 ms). The red transparent box indicates time of sound pulse stimulation. $\boldsymbol{d}$, Group PSTHs showing increased Z score for basolateral neurons recorded during unpredictably (red bars) versus predictably timed (blue bars) sound pulse stimulation (bins of $20 \mathrm{~ms} ; n=$ $28 ; p=0.007,2$-tailed paired $t$ test on the average $Z$ score $0-80 \mathrm{~ms}$ after sound pulse onset). The red box indicates time of sound pulse stimulation. $\boldsymbol{e}$, Sound pulse-evoked neural responses (plotted as the percentage of the $Z$ score of the first 2 sound pulses) during predictable (blue symbols) and unpredictable (red symbols) sound pulse sequence stimulation. During predictably timed but not during unpredictably timed sound pulse stimulation, neural responses exhibit rapid and significant habituation (predictable: $F_{(27,4)}=2.83, p=0.03$; unpredictable: $F_{(27,4)}=0.17, p=0.95 ; 1$-way ANOVA with repeated measures on the average $Z$ score $0-80 \mathrm{~ms}$ after sound pulse onset). Pred., Predictably timed; Unpred., unpredictably timed.

ably timed sound pulses was used. We compared the blood oxygen level-dependent (BOLD) signal response using GLM contrasts and random-effects analyses to examine stationary differential BOLD activity related to stimulus conditions. As a main effect, both predictable and unpredictable sound pulse sequence stimulation produced BOLD activity in bilateral auditory cortex (Talairach peak $x / y / z$ coordinates: left side, $-36 /-16 /+7 \mathrm{~mm}$, $t_{(19)}=11.7, p<0.05$ after Bonferroni correction; right side, $+42 /-16 /+7 \mathrm{~mm}, t_{(19)}=11.9, p<0.05$ after Bonferroni correction). The comparison of brain activity, using linear contrast analysis of BOLD responses, yielded significant clusters bilaterally in amygdala in response to sequences with unpredictably compared with predictably timed sound pulses (Fig. 5a) (Talairach peak $x / y / z$ coordinates: left side, $-19 /-10 /-14 \mathrm{~mm}$, $t_{(19)}=5.9, p=0.00001$; right side, $+24 /-16 /-17 \mathrm{~mm}, t_{(19)}=$ $4.7, p<0.0002)$. In addition, the rather posterior location of the peak Talairach coordinates, especially on the right side, indicates activation of the adjacent anterior hippocampus (Krabbendam et al., 2000). Notably, no differential activity was found in auditory cortex, auditory thalamus, prefrontal cortex, or any other brain region studied, which supports the notion that the activation of amygdala was not an unspecific effect of unpredictable stimuli. The amygdala typically habituates rapidly to prolonged stimulation (Breiter et al., 1996; Büchel et al., 1998; LaBar et al., 1998); thus, we finally performed a region-of-interest-based analysis, which yielded that the detected effects of unpredictably timed sound pulses in amygdala were persistent across the entire stimulation period of $60 \mathrm{~s}$ and were not driven by transient responses or habituation effects. This demonstrates that, like in rodents, temporal unpredictability induces sustained activation of human amygdala.

\section{Unpredictability enhances spatial attention toward emotional face expressions}

Because sequences of unpredictably timed sound pulses induced anxiety-like behavior in mice, we examined behavioral consequences in healthy humans. First, we presented subjects with sequences of predictably and unpredictably timed sound pulses and asked to rate which sequence they found more unpleasant. Of 40 subjects examined, 23 subjects found unpredictable sound pulse sequence stimulation more unpleasant than stimulation with predictable sound pulse sequences $\left(\chi^{2}=\right.$ $0.9 ; p=0.34)$. This indicates that unpredictable sound pulse sequences were generally not perceived as more unpleasant than predictable sound pulse sequences. Then, we examined the attention toward threat information, which is known to be increased in subjects with trait anxiety and anxiety disorders (Mogg et al., 1997; Bradley et al., 1999). In a dot-probe paradigm, subjects typically respond faster to dots that appear in the place of threat stimuli (congruent trials) compared with dots that appear in other spatial locations (incongruent trials). This anisotropy represents a measure for spatial attention toward threat stimuli and is quantified by the so-called bias index (defined as RT difference between congruent and incongruent trials). Here, we used angry faces (Schmidt and Erdmann, 2003) as threat stimuli because of the central role of the amygdala in processing emotional face stimuli (Breiter et al., 1996; Anderson and Phelps, 2001; Whalen et al., 2004; Adolphs et al., 2005) (Fig. 5b). We found an increase of the bias index during sequences of unpredictably compared with predictably timed sound pulses $\left(F_{(1,34)}=7.04 ; p=0.01\right)$ (Fig. $\left.5 c\right)$; this effect was even more pronounced in subjects with longer RTs in neutral trials $\left(F_{(1,34)}=7.82 ; p<0.01\right)$. Consistent with previous studies, the unpredictability-related bias index increase was mainly caused by RT prolongations in incongruent trials, which has been described as difficulty to disengage from threat stimuli (Koster et al., 2004). Thus, consistent with a role for the amygdala in processing emotional face expressions in humans, unpredictability- 
a

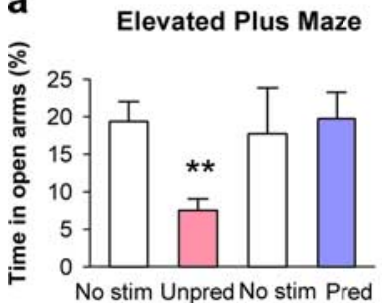

b

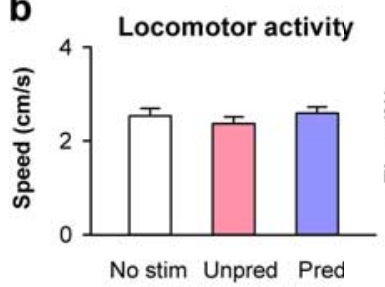

C
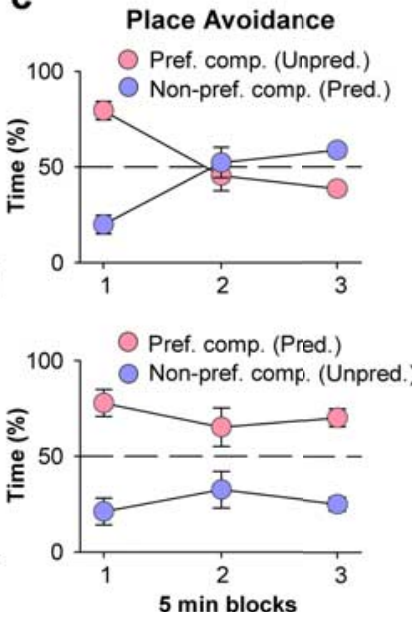

Figure 4. Unpredictably timed sound pulse sequence stimulation induces anxiety-like and avoidance behavior in mice. $\boldsymbol{a}$, Unpredictably timed sound pulse stimulation decreases time spent on open arms of the EPM (unpredictable vs silent control period: $n=5, p=0.005$, paired $t$ test; predictable vs silent control period: $n=5, p=0.649$, paired $t$ test; unpredictable vs predictable: $n=5$ per group, $p=0.021$, unpaired $t$ test; ${ }^{* *} p<0.01$ ). $\boldsymbol{b}$, No difference in locomotor activity was measured by the mean speed (centimeters per second) during unpredictably and predictably timed sound pulse stimulation (unpredictable vs silent control period: $n=8, p=0.246$, paired $t$ test; predictable vs silent control period: $n=8, p=0.704$, paired $t$ test; unpredictable vs predictable: $n=8, p=0.205$, paired $t$ test; $\left.{ }^{* *} p<0.01\right)$. c, Top, Unpredictably timed sound pulse stimulation induces avoidance behavior in a two-compartment place-aversion paradigm. Systematic exposure to unpredictably timed sound pulse stimulation in the preferred compartment (and predictably timed stimulation in the nonpreferred compartment) leads to progressive avoidance of the preferred compartment $\left[n=5 ; F_{(2,16)}=39.82\right.$; $p<0.001$, 2-way ANOVA with repeated measures (interaction between group $\times$ time point)]. Direct comparison between predictably and unpredictably timed sound pulse stimulations shows a significant difference for the first and the last time points $\left(F_{(1,8)}=60.04, p<0.001\right.$ and $F_{(1,8)}=16.37, p<0.01$, Scheffé's $F$ test). Bottom, Same as top panel, except that during training, unpredictably timed sound pulse stimulation was delivered in the nonpreferred compartment $(n=6)$. Mice continued to spend more time in the preferred compartment if it was associated with predictably timed sound pulses $\left[F_{(2,20)}=1.57, p=0.23\right.$, 2-way ANOVA with repeated measures (interaction between group $X$ time point)]. No stim., No stimulation; Unpred., unpredictably timed; Pred., predictably timed; Pref. comp., preferred compartment; Nonpref. comp., nonpreferred compartment.

induced sustained amygdala activation is associated with enhanced spatial attention bias toward angry compared with neutral faces.

\section{Discussion}

Our results show that unpredictable auditory stimulation is sufficient to induce sustained neural activity in the amygdala and to elicit anxiety-like behavior in mice and humans. In mice, temporal unpredictability induced an increase in the expression of the activity-dependent neuronal immediate-early gene c-fos, prevented habituation of single-cell activity within the BLA, and increased avoidance and anxiety-like behavior. Likewise, in humans, temporal unpredictability induced sustained neural activity in the amygdala as revealed by fMRI and promoted anxietylike behavior as quantified by enhanced attention toward emotional faces. These results indicate that habituation of neuronal activity in the amygdala represents an evolutionary conserved mechanism by which temporal predictability of perceptual input leads to adaptive changes in emotional behavior.

In mice, the amygdala was the only brain region in which we found more activity during unpredictably than during predictably timed auditory stimulation. Whereas our data do not allow to exclude the contribution of dedicated neural circuits in other
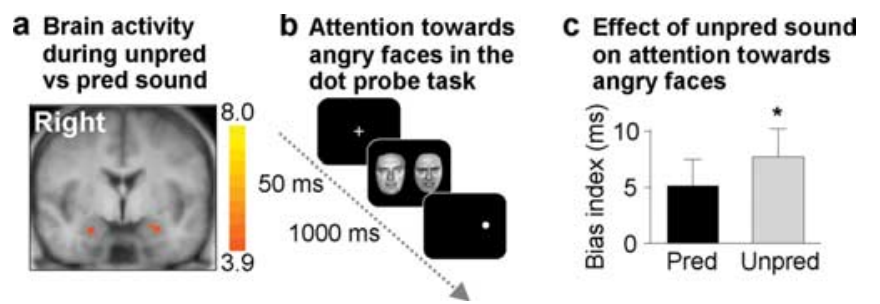

Figure 5. Effects of auditory stimulation with sequence of unpredictably timed sound pulses on human brain activity and behavior. $\boldsymbol{a}$, fMRI yields sustained amygdala activity during $60 \mathrm{~s}$ stimulation with a sequence of temporally unpredictable compared with predictable sound pulses. Shown is a coronal slice ( $y,-11 \mathrm{~mm}$ ) of Talairach-transformed anatomical MRI brain scan (averaged across all subjects) with a statistical parametric map superimposed [balanced random-effects GLM contrast; voxel-level threshold, $p_{\text {uncorrected }}<0.001$; cluster-level threshold $\left(111 \mathrm{~mm}^{3}\right), p_{\text {corrected }}<0.05$; color gradient, $t_{(19)}$ values $]$. $\boldsymbol{b}$, Timeline of visual dot-probe task measuring attention toward threat information. The task was performed during presentation of sequences of predictably and unpredictably timed sound pulses. After the presentation of a fixation cross ( $950 \mathrm{~ms}$ ) and two modified Ekman faces (Büchel et al., 1998), subjects had to respond by key press to the position of a dot appearing at the position of one of the two faces. $\boldsymbol{C}$, The bias index served as a quantitative measure of attention toward threat information in the visual dot probe; it was calculated as RT difference between trials with the dot appearing in the angry face's position (congruent) and those with the dot in the opposite position (incongruent). The presentation of sequences with unpredictable sound pulse timing during the dot probe task produced an increase in bias index (mean $\pm \mathrm{SEM} ;{ }^{*} F_{(1,34)}=7.04 ; p=0.01$ ), indicating unpredictability-related enhanced (anxiety-like) attention toward angry faces. Unpred, Unpredictably timed; Pred, predictably timed.

brain areas, the activity of which could have escaped our detection methods, the most parsimonious interpretation suggests that detection of temporal unpredictability occurs in the amygdala. Based on recent results suggesting that the integrity of the amygdala is necessary for place-avoidance learning (Holahan and White, 2004), it is likely that unpredictability-induced amygdala activation promotes avoidance behavior. Interestingly, it has been shown in mice that predictable footshocks given in a particular context promote preference for this context. In contrast, mice tend systematically to avoid a context previously paired with unpredictable footshocks (Orsini et al., 2002). Our present results extend these findings by demonstrating that unpredictability per se without interaction with motivational information is sufficient to elicit avoidance behavior.

Although unpredictably timed auditory stimulation induced greater amygdala activity than predictably timed stimulation, amygdala neurons were significantly activated during predictable auditory stimulation. This was, however, not associated with any anxiety-like or avoidance behavior, suggesting that the rapid habituation of neuronal activity during exposure to predictable stimulation patterns keeps activation levels below the behaviorally relevant threshold or induces very rapid behavioral habituation. Consistent with this idea, previous studies have shown that various forms of behavioral habituation occur more rapidly if sensory stimuli are delivered at constant rather than variable interstimulus intervals (McDaniel and White, 1966; Mackworth, 1968; Davis, 1970). Most of these studies, however, have used interstimulus intervals at least one order of magnitude longer than those used here, making it difficult to directly compare the results.

It remains an open question how neural circuits in the amygdala rapidly adapt to predictable stimulation and why this does not occur if auditory stimuli are delivered in an unpredictably timed manner. Previous studies suggested that habituating and nonhabituating BLA neurons might represent different types of cells (Ben-Ari and Le Galle La Salle, 1974; Bordi and LeDoux, 1992). Although our results certainly do not exclude the existence 
of distinct nonhabituating types of neurons in the BLA, they demonstrate that a given neuron can switch between habituating and nonhabituating states depending on the temporal structure of sensory stimulation. Because changes in spontaneous activity only marginally contribute to the observed effect, neuromodulation of cellular excitability appears unlikely to be the major mechanism underlying differential habituation. Modulation of mechanisms implicated in the adaptation of synaptic transmission at extrinsic afferents or within local amygdala circuitry may represent possible ways to shift a neuron from a habituating to a nonhabituating state (Grill-Spector et al., 2006). Alternatively, recruitment of inhibitory networks within the amygdala may actively suppress principal cell activity leading to habituation of neuronal responses (Lang and Paré, 1997).

Conceptions of unpredictability in previous studies in humans have often included aversive and potentially noxious events such as electric shocks or pain (Chan and Lovibond, 1996; Bornhovd et al., 2002; Grillon, 2002; Grillon et al., 2004). In such paradigms, unpredictability obviates periods of safety from negative events, the consequences of which, however, must constantly be anticipated. Therefore, these paradigms measure unsafety (Seligman and Binik, 1977) (i.e., constant anticipation of noxious consequences rather than the effect of unpredictability per se). Unpredictability of neutral events has been examined in the context of deviant events and local entropy variation (Fletcher et al., 2001; Strange et al., 2005). These paradigms are not characterized by the absence of predictable patterns but rather by small deviances from existing patterns, which require working memory resources to be detected. Because in all of these paradigms salient patterns are present, they examine the consequences of pattern deviation, which does not seem to be mediated by the amygdala (Fletcher et al., 2001; Ulanovsky et al., 2003; Strange et al., 2005). Thus, temporal unpredictability is processed similar to ambiguity, defined as the degree to which consequences of a forthcoming event cannot be foreseen (Hsu et al., 2005) or, in more general terms, as a state of persistent novelty (Rutishauser et al., 2006).

In contrast to animals, unpredictably timed auditory stimulation also activated the anterior hippocampus in humans. Previous studies have implicated the hippocampus in behavioral control during anxiety states (Gray and McNaughton, 2000). Moreover, hippocampal activation correlates with the active cognitive detection of varying unpredictability in complex stimulus series (Strange et al., 2005). The reason why temporal unpredictability leads to a differential engagement of the anterior (ventral) hippocampus in humans and mice remains unclear but might reflect differential cognitive processing of temporal unpredictability.

In general, screening the sensory environment for threat and reward is an adaptive operation an organism constantly performs. Cues and, in more general terms, patterns help to reduce the complexity of sensory information by creating concepts and templates that can be used to evaluate salience in the sensory environment and thus detect potential danger. Hence, organisms have a propensity to identify patterns even in an environment that consists merely of random information as soon as regularities occur (Nickerson, 2002). If no temporal pattern exists, attempts to predict the temporal structure of a sensory stimulus is bound to generate prediction errors, which are classically thought to act as feedback-teaching signals during various learning paradigms (Schultz and Dickinson, 2000). Thus, given the extensive literature demonstrating that amygdala activity can facilitate memory consolidation in other brain structures including neocortex and hippocampus (McGaugh, 2004), sustained amygdala activity induced by exposure to unpredictable auditory stimuli may represent one possible mechanism by which prediction errors generated in one brain area may influence more widespread memory systems.

Finally, behavioral changes and alterations in amygdala activity similar to those induced by unpredictability are associated with anxiety traits or anxiety disorders in humans (Mogg et al., 1997; Bradley et al., 1999; Etkin et al., 2004). Thus, our study not only identifies temporal unpredictability as an important feature of the sensory environment that influences emotional behavior but also provides a translational model for studying the neural mechanisms underlying sustained amygdala activation associated with psychiatric conditions.

\section{References}

Adolphs R, Damasio H, Tranel D, Damasio AR (1996) Cortical systems for the recognition of emotion in facial expressions. J Neurosci 16:7678-7687.

Adolphs R, Gosselin F, Buchanan TW, Tranel D, Schyns P, Damasio AR (2005) A mechanism for impaired fear recognition after amygdala damage. Nature 433:68-72.

Anderson AK, Phelps EA (2001) Lesions of the human amygdala impair enhanced perception of emotionally salient events. Nature 411:305-309.

Becerra L, Breiter HC, Wise R, Gonzalez RG, Borsook D (2001) Reward circuitry activation by noxious thermal stimuli. Neuron 32:927-946.

Ben-Ari Y, Le Gal La Salle G (1974) Lateral amygdala unit activity: II. Habituating and non-habituating neurons. Electroencephalogr Clin Neurophysiol 37:463-472.

Bisler S, Schleicher A, Gass P, Stehle JH, Zilles K, Staiger JF (2002) Expression of c-Fos, ICER, Krox-24 and JunB in the whisker-to-barrel pathway of rats: time course of induction upon whisker stimulation by tactile exploration of an enriched environment. J Chem Neuroanat 23:187-198.

Bordi F, LeDoux J (1992) Sensory tuning beyond the sensory system: an initial analysis of auditory response properties of neurons in the lateral amygdaloid nucleus and overlying areas of the striatum. J Neurosci 12:2493-2503.

Bornhovd K, Quante M, Glauche V, Bromm B, Weiller C, Büchel C (2002) Painful stimuli evoke different stimulus-response functions in the amygdala, prefrontal, insula and somatosensory cortex: a single-trial fMRI study. Brain 125:1326-1336.

Boynton GM, Engel SA, Glover GH, Heeger DJ (1996) Linear systems analysis of functional magnetic resonance imaging in human V1. J Neurosci 16:4207-4221.

Bradley BP, Mogg K, White J, Groom C, de Bono J (1999) Attentional bias for emotional faces in generalized anxiety disorder. Br J Clin Psychol 38:267-278.

Breiter HC, Etcoff NL, Whalen PJ, Kennedy WA, Rauch SL, Buckner RL, Strauss MM, Hyman SE, Rosen BR (1996) Response and habituation of the human amygdala during visual processing of facial expression. Neuron 17:875-887.

Büchel C, Dolan RJ (2000) Classical fear conditioning in functional neuroimaging. Curr Opin Neurobiol 10:219-223.

Büchel C, Morris J, Dolan RJ, Friston KJ (1998) Brain systems mediating aversive conditioning: an event-related fMRI study. Neuron 20:947-957.

Bullmore E, Brammer M, Williams SC, Rabe-Hesketh S, Janot N, David A, Mellers J, Howard R, Sham P (1996) Statistical methods of estimation and inference for functional MR image analysis. Magn Reson Med 35:261-277.

Chan CK, Lovibond PF (1996) Expectancy bias in trait anxiety. J Abnorm Psychol 105:637-647.

Chaudhuri A, Zangenehpour S, Rahbar-Dehgan F, Ye F (2000) Molecular maps of neural activity and quiescence. Acta Neurobiol Exp 60:403-410.

Davis M (1970) Effects of interstimulus interval length and variability on startle-response habituation in the rat. J Comp Physiol Psychol 72:177-192.

Davis M, Whalen PJ (2001) The amygdala: vigilance and emotion. Mol Psychiatry 6:13-34.

Ekman P, Friesen W (1975) Pictures of facial affect. Palo Alto, CA: Consulting Psychologists. 
Etkin A, Klemenhagen KC, Dudman JT, Rogan MT, Hen R, Kandel ER, Hirsch J (2004) Individual differences in trait anxiety predict the response of the basolateral amygdala to unconsciously processed fearful faces. Neuron 44:1043-1055.

Filipkowski RK, Rydz M, Berdel B, Morys J, Kaczmarek L (2000) Tactile experience induces $\mathrm{c}$-fos expression in rat barrel cortex. Learn Mem 7:116-122.

Fiorillo CD, Tobler PN, Schultz W (2003) Discrete coding of reward probability and uncertainty by dopamine neurons. Science 299:1898-1902.

Fletcher PC, Anderson JM, Shanks DR, Honey R, Carpenter TA, Donovan T, Papadakis N, Bullmore ET (2001) Responses of human frontal cortex to surprising events are predicted by formal associative learning theory. Nat Neurosci 4:1043-1048.

Forman SD, Cohen JD, Fitzgerald M, Eddy WF, Mintun MA, Noll DC (1995) Improved assessment of significant activation in functional magnetic resonance imaging (fMRI): use of a cluster-size threshold. Magn Reson Med 33:636-647.

Franklin KJB, Paxinos G (1997) The mouse brain in stereotaxic coordinates. New York: Academic.

Friston KJ, Holmes AP, Worsley KJ, Poline JP, Frith CD, Frackowiak RSJ (1995) Statistical parametric maps in functional imaging-A general linear approach. Hum Brain Mapp 2:189-210.

Gray JA, McNaughton N (2000) The neuropsychology of anxiety: an enquiry into the functions of the septo-hippocampal system. Oxford: Oxford UP.

Grill-Spector K, Henson R, Martin A (2006) Repetition and the brain: neural models of stimulus-specific effects. Trends Cog Sci 10:14-23.

Grillon C (2002) Associative learning deficits increase symptoms of anxiety in humans. Biol Psychiatry 51:851-858.

Grillon C, Baas JP, Lissek S, Smith K, Milstein J (2004) Anxious responses to predictable and unpredictable aversive events. Behav Neurosci 118:916-924.

Grillon C, Baas JM, Cornwell B, Johnson L (2006) Context conditioning and behavioral avoidance in a virtual reality environment: effect of predictability. Biol Psychiatry 60:752-759.

Herry C, Mons N (2004) Resistance to extinction is associated with impaired immediate early gene induction in medial prefrontal cortex and amygdala. Eur J Neurosci 20:781-790.

Holahan MR, White NM (2004) Intra-amygdala muscimol injections impair freezing and place avoidance in aversive contextual conditioning. Learn Mem 11:436-446.

Holland PC, Gallagher M (1993) Amygdala central nucleus lesions disrupt increments, but not decrements, in conditioned stimulus processing. Behav Neurosci 107:246-253.

Hsu M, Bhatt M, Adolphs R, Tranel D, Camerer CF (2005) Neural systems responding to degrees of uncertainty in human decision-making. Science 310:1680-1683.

Koster EH, Crombez G, Verschuere B, De Houwer J (2004) Selective attention to threat in the dot probe paradigm: differentiating vigilance and difficulty to disengage. Behav Res Ther 42:1183-1192.

Krabbendam L, Derix M, Honig A, Vuurman E, Havermans R, Wilmink JT, Jolles J (2000) Cognitive performance in relation to MRI temporal lobe volume in schizophrenic patients and healthy control subjects. J Neuropsychiat Clin Neurosci 12:251-256.

LaBar KS, Gatenby JC, Gore JC, LeDoux JE, Phelps EA (1998) Human amygdala activation during conditioned fear acquisition and extinction: a mixed-trial fMRI study. Neuron 20:937-945.

Lang EJ, Paré D (1997) Similar inhibitory processes dominate the responses of cat lateral amygdaloid projection neurons to their various afferents. J Neurophysiol 77:341-352.

LeDoux JE (2000) Emotion circuits in the brain. Annu Rev Neurosci 23:155-184.
Lister RG (1987) The use of a plus-maze to measure anxiety in the mouse. Psychopharmacology (Berl) 92:180-185.

Mackworth JF (1968) Vigilance and habituation. Psychol Rev 75:308-322.

McDaniel JW, White RK (1966) A factorial study of the stimulus conditions of habituation. Percept Mot Skills 23:259-270.

McGaugh JL (2004) The amygdala modulates the consolidation of memories of emotionally arousing experiences. Annu Rev Neurosci 27:1-28.

Mineka S, Kihlstrom JF (1978) Unpredictable and uncontrollable events: a new perspective on experimental neurosis. J Abnorm Psychol $87: 256-271$.

Mogg K, Bradley BP, de Bono J, Painter M (1997) Time course of attentional bias for threat information in non-clinical anxiety. Behav Res Ther 35:297-303.

Mogg K, McNamara J, Powys M, Rawlinson H, Seiffer A, Bradley BP (2000) Selective attention to threat: a test of two cognitive models of anxiety. Cogn Emotion 14:375-399.

Nickerson RS (2002) The production and perception of randomness. Psychol Rev 109:330-357.

Orsini C, Ventura R, Lucchese F, Puglisi-Allegra S, Cabib S (2002) Predictable stress promotes place preference and low mesoaccumbens dopamine response. Physiol Behav 75:135-141.

Paz R, Pelletier JG, Bauer EP, Paré D (2006) Emotional enhancement of memory via amygdala-driven facilitation of rhinal interactions. Nat Neurosci 9:1321-1329.

Phelps EA, LeDoux JE (2005) Contributions of the amygdala to emotion processing: from animal models to human behavior. Neuron 48:175-187.

Quirk GJ, Armony JL, LeDoux JE (1997) Fear conditioning enhances different components of tone-evoked spike trains in auditory cortex and lateral amygdala. Neuron 19:613-624.

Rosen JB, Donley MP (2006) Animal studies of amygdala function in fear and uncertainty: relevance to human research. Biol Psychol 73:49-60.

Rutishauser U, Mamelak AN, Schuman EM (2006) Single-trial learning of novel stimuli by individual neurons of the human hippocampusamygdala complex. Neuron 49:805-813.

Schmahl C, Bohus M, Esposito F, Treede RD, Di Salle F, Greffrath W, Ludaescher P, Jochims A, Lieb K, Scheffler K, Hennig J, Seifritz E (2006) Neural correlates of antinociception in borderline personality disorder. Arch Gen Psychiatry 63:659-667.

Schmidt M, Erdmann G (2003) Development and test of a modified facein-the-crowd task. J Psychophysiol 17:177.

Schultz W, Dickinson A (2000) Neural coding of prediction errors. Annu Rev Neurosci 23:473-500.

Seligman ME, Binik YM (1977) The safety-signal hypothesis. In: OperantPavlovian interactions (Davis H, Hurwitz HMB, eds), pp 165-187. Hillsdale, NJ: Erlbaum.

Strange BA, Duggins A, Penny W, Dolan RJ, Friston KJ (2005) Information theory, novelty and hippocampal responses: unpredicted or unpredictable? Neural Netw 18:225-230.

Ulanovsky N, Las L, Nelken I (2003) Processing of low-probability sounds by cortical neurons. Nat Neurosci 6:391-398.

Vyas A, Chattarji S (2004) Modulation of different states of anxiety-like behavior by chronic stress. Behav Neurosci 118:1450-1454.

Weiskrantz L (1956) Behavioral changes associated with ablation of the amygdaloid complex in monkeys. J Comp Physiol Psychol 49:381-391.

Whalen PJ (1998) Fear, vigilance, and ambiguity: initial neuroimaging studies of the human amygdala. Curr Dir Psychol Sci 7:177-188.

Whalen PJ, Kagan J, Cook RG, Davis FC, Kim H, Polis S, McLaren DG, Somerville LH, McLean AA, Maxwell JS, Johnstone T (2004) Human amygdala responsivity to masked fearful eye whites. Science 306:2061.

Whalen PJ, Shin LM, McInerney SC, Fischer H, Wright CI, Rauch SL (2001) A functional MRI study of human amygdala responses to facial expressions of fear versus anger. Emotion 1:70-83. 